\title{
Surface Electrochemistry: Structured Electrode, Synthesis, and Characterization
}

\author{
Fethi Bedioui, ${ }^{1}$ Tebello Nyokong, ${ }^{2}$ and José H. Zagal ${ }^{3}$ \\ ${ }^{1}$ Unité de Pharmacologie Chimique et Génétique et Imagerie, UMR CNRS 8151-U INSERM 1022, \\ Chimie ParisTech and Université Paris Descartes, 11 rue Pierre et Marie Curie, 75231 Paris, France \\ ${ }^{2}$ Department of Chemistry, Rhodes University, Grahamstown 6140, South Africa \\ ${ }^{3}$ Departamento de Química de los Materiales, Facultad de Química y Biología, Universidad de Santiago de Chile, Casilla 40, \\ Sucursal Matucana, Santiago 9170022, Chile
}

Correspondence should be addressed to Fethi Bedioui, fethi-bedioui@chimie-paristech.fr

Received 1 December 2011; Accepted 1 December 2011

Copyright (C) 2012 Fethi Bedioui et al. This is an open access article distributed under the Creative Commons Attribution License, which permits unrestricted use, distribution, and reproduction in any medium, provided the original work is properly cited.

Electrochemistry has become a multidisciplinary science, and a growing emphasis in the last decades has been focused on the chemical control of the structure of the electrode/ solution interface in order to achieve desired reactivity for electron transfer processes involving target molecules. Thus, modified electrode surfaces have become a wide topic that covers several issues of interfacial electrochemistry, nanotechnology, material science, sensors for biology and environmental sciences, and so forth.

The aim of this special issue is to show, through recent updated significant examples, how the electrochemical techniques allow the unique characterization of specific properties of micro- and nanostructured materials that offer varied possibilities of uses and the preparation of specific types of ordered materials that take advantage of electrochemical synthetic methods such as structuring nanosized wires and dots, to cite only two examples.

Five original research articles are presented in this issue and illustrate some of the efforts in developing electrochemical strategies related to structured surfaces. Three themes are concerned: electrochemical analysis of electrode surfaces upon specific chemical treatments, electrochemical deposition of structured metal deposits and electrochemical characterization of polymer-coated electrodes. In the paper entitled "Surface of alumina films after prolonged breakdowns in galvanostatic anodization" by C. Girginov and S. Kozhukharov, the authors investigated the breakdown phenomena at continuous isothermal $\left(20^{\circ} \mathrm{C}\right)$ and galvanostatic $\left(0.2-5 \mathrm{~mA} \mathrm{~cm}^{-2}\right)$ anodizing of aluminum in ammonium salicylate in dimethylformamide (1 M AS/DMF) electrolyte.
Data on topography and surface roughness parameters of the electrode after electric breakdowns are obtained as a function of anodization time. The results are discussed on the basis of perceptions of avalanche mechanism of the breakdown phenomena, due to the injection of electrons and their multiplication in the volume of the film.

In the paper entitled "Surface potential of polycrystaline hematite in aqueous medium" by T. Preočanin et al., the surface potential of polycrystalline hematite in aqueous sodium perchlorate environment as a function of $\mathrm{pH}$ was examined. Surface potential of hematite was obtained from measured electrode potential of a nonporous polycrystalline hematite electrode, and interpretation of the equilibrium data was performed by applying the surface complexation model.

In the paper entitled "Zinc-nickel codeposition in sulfate solution combined effect of cadmium and boric acid" by Y. Addia and A. Khouider, the combined effect of cadmium and boric acid on the electrodeposition of zinc-nickel from a sulfate has been investigated. It is shown that the presence of cadmium ion decreases zinc in the deposit. Low concentration of $\mathrm{CdSO}_{4}$ reduces the anomalous nature of $\mathrm{Zn}-\mathrm{Ni}$ deposit while boric acid decreases current density and shifts potential discharge of nickel and hydrogen to more negative potential.

In the paper entitled "Size and shape control of gold nanodeposits in an array of silica nanowells on a gold electrode" by A. E. Rue and M. M. Collinson, ordered arrays of hemispherical nanowells were formed in a sol-gel derived silica film on a gold electrode using $500 \mathrm{~nm}$ diameter polystyrene latex spheres as templates. The conductive domain located 
at the bottom of each nanowell upon template removal was enlarged via electroless deposition from a gold plating solution. The structured electrodes thus formed were characterized using scanning electron microscopy and atomic force microscopy. Electroless deposition in the nanowells produced (near) sphere-like nanostructures of gold, the size of which depended on the incubation time in the plating solution and the size of the conductive domain. Longer exposure times yielded nanostructures that filled the nanowell whereas smaller exposure time yielded much smaller structures. Significantly larger, rougher deposits were formed in nanowells with large conductive domains. The electrochemical response observed at these electrodes was strongly dependent on the extent of long-range packing, the presence of defect sites in the film and their relative spacing, and the redox species in solution.

Finally, in the paper entitled "Cyclic voltammetry and impedance spectroscopy behavior studies of polyterthiophene modified electrode" by N. Maouche and B. Nessark, a study of the electrochemical behaviour of terthiophene and its corresponding polymer, which is obtained electrochemically as a film by cyclic voltammetry (CV) on platinum electrode, is presented. The analysis focuses essentially on the effect of two organic solvents acetonitrile and dichloromethane on the electrochemical behaviour of the obtained polymer. The electrochemical behavior of this material was investigated by cyclic voltammetry and electrochemical impedance spectroscopy (EIS). The impedance plots show the semicircle which is characteristic of charge transfer resistance at the electrode/polymer interface at high frequency and the diffusion process at low frequency.

Fethi Bedioui

Tebello Nyokong

José H. Zagal 


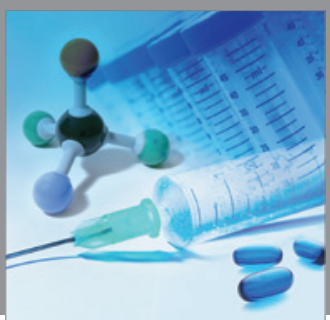

International Journal of

Medicinal Chemistry

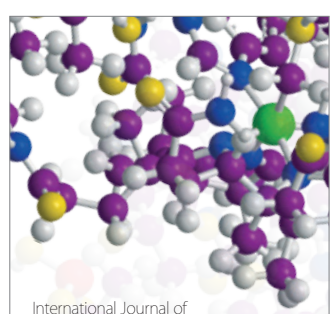

Carbohydrate Chemistry

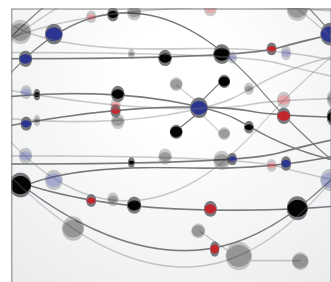

The Scientific World Journal
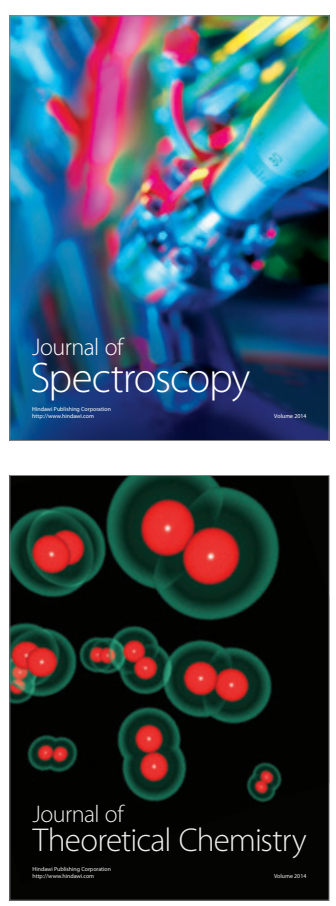
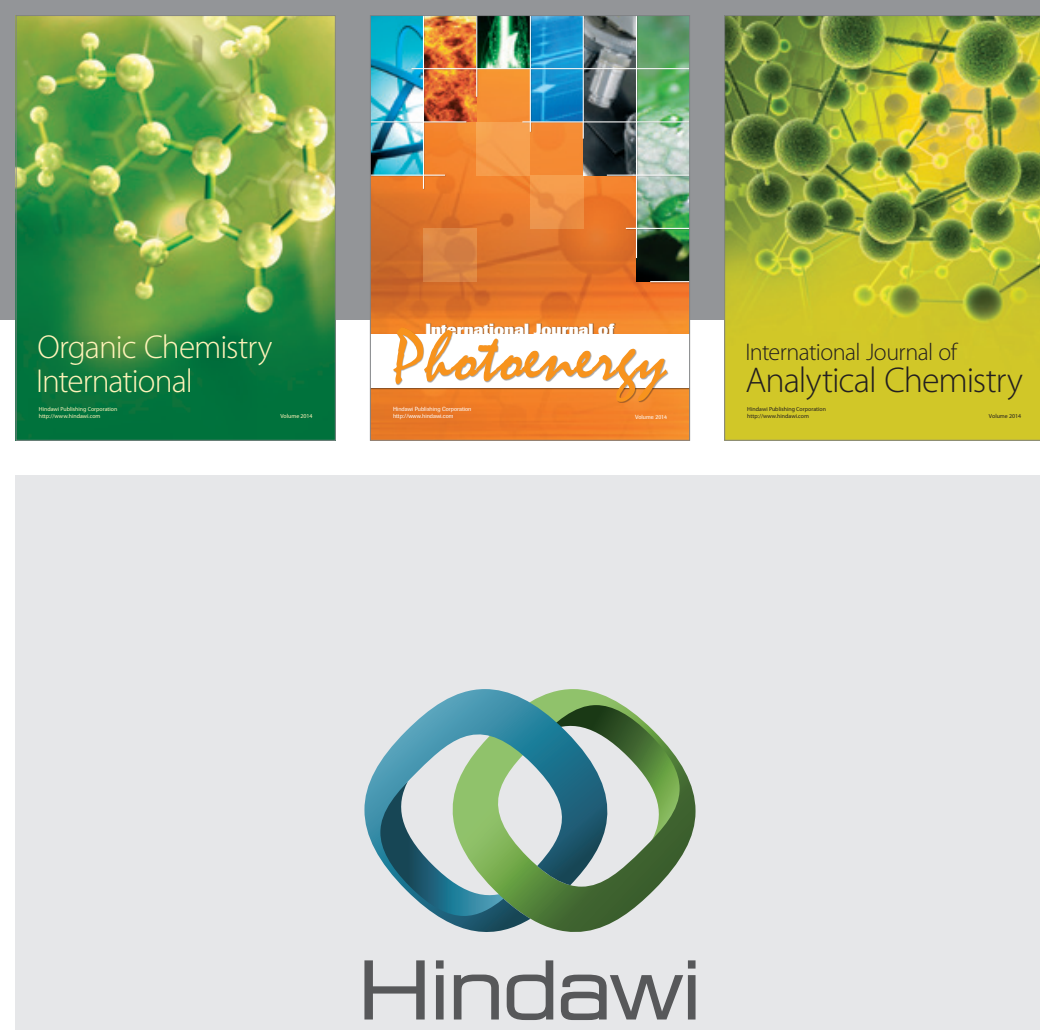

Submit your manuscripts at

http://www.hindawi.com
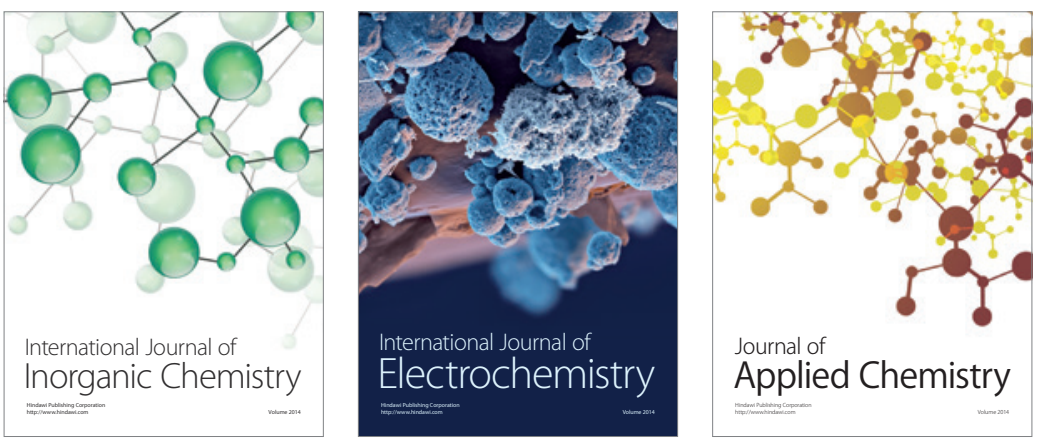

Journal of

Applied Chemistry
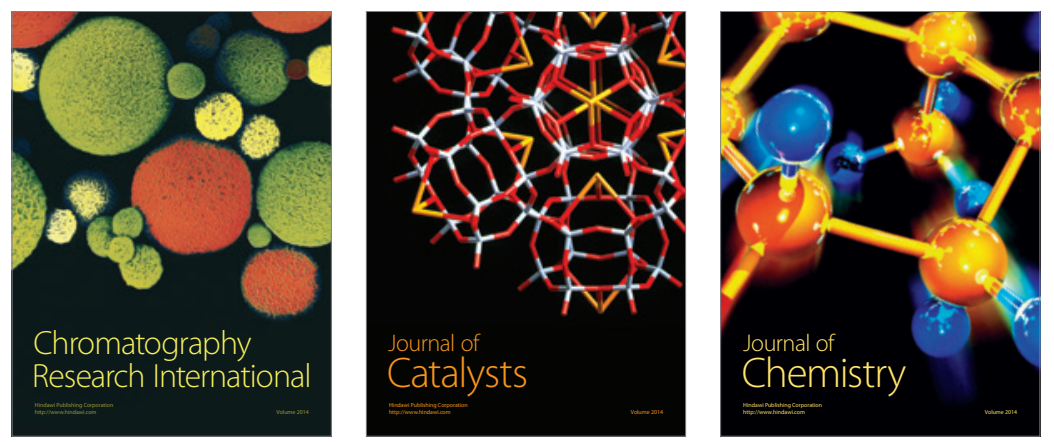
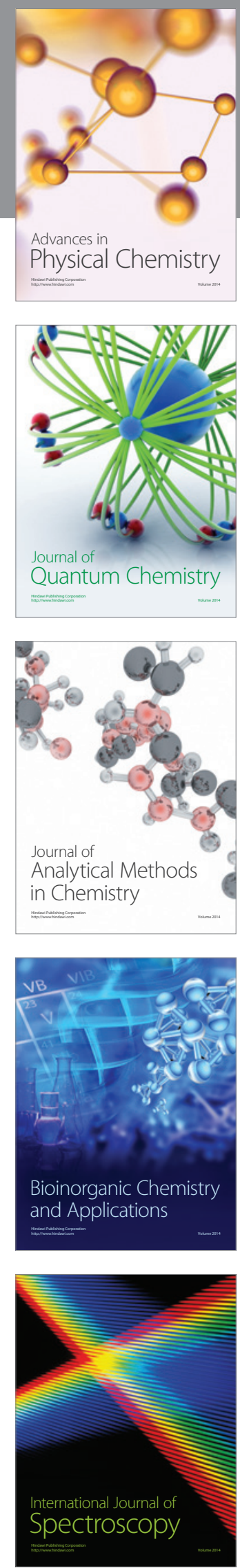